\title{
(2) OPEN ACCESS \\ Mid-arm circumference can be used to estimate weight of adult and adolescent patients
}

\author{
Giles N Cattermole, ${ }^{1}$ Colin A Graham, ${ }^{2}$ Timothy H Rainer ${ }^{3}$
}

\begin{abstract}
- Additional material is published online only. To view please visit the journal online (http://dx.doi.org/10.1136/ emermed-2015-205623).
\end{abstract}

'Emergency Department, Centre Hospitalier Universitaire de Kigali, Kigali, Rwanda

${ }^{2}$ Accident and Emergency Medicine Academic Unit, Chinese University of Hong Kong, Shatin, Hong Kong 3Emergency Department, University Hospital of Wales, Cardiff, UK

Correspondence to Dr Giles N Cattermole Emergency Department, Centre Hospitalier Universitaire de Kigali, Kigali, Rwanda; giles@ cattermole.org.uk

Received 10 December 2015 Revised 29 October 2016 Accepted 24 November 2016 Published Online First 19 December 2016

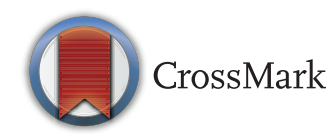

To cite: Cattermole GN, Graham CA, Rainer TH. Emerg Med J 2017;34:231236

\section{ABSTRACT}

Objectives Many drug and fluid regimens in emergency medicine are weight dependent in adults, but no standard adult weight estimation tools exist. Paediatric weight is often estimated in emergency situations using methods based on age or height when direct measurement is not possible, and recently, methods based on mid-arm circumference (MAC) have also been developed. The aim of this study was to derive and validate an accurate MAC-based method for weight estimation for use in all age groups.

Methods Data were obtained from the US National Health and Nutrition Examination Survey (NHANES). MAC-based methods of weight estimation were derived in 8498 subjects (5595 adults aged $16-80$ years, 2903 children aged 1-15.9 years) from the NHANES 2011-2012 dataset, using linear regression. NHANES 2009-2010 was used for validation in 9022 subjects (6049 adults aged 16-79 years, 2973 children aged 1-15.9 years).

Results A simplified method of MAC-based weight estimation was derived from linear regression equation: weight in $\mathrm{kg}=4 \times \mathrm{MAC}$ (in $\mathrm{cm}$ ) - 50 . On validation, results in children aged 1-10.9 years were poor. In adults and children aged $11-15.9$ years, over $60 \%$, $90 \%$ and $98 \%$ of estimates fell, respectively, within $10 \%, 20 \%$ and $30 \%$ of actual weights when using the simplified formula.

Conclusions In this description of a method for estimating weight in adults, we have derived and validated a simplified formula that is at least as precise in adults and adolescents as commonly used paediatric weight estimation tools in children.

\section{INTRODUCTION}

In adult medicine, it is common practice (although unlikely to be good practice) for many drugs to be given as a single adult dose, whether the patient weighs 50 or $100 \mathrm{~kg}$. However, many drugs used in time-critical situations do require more precise dosing according to weight, for example, local anaesthetics, induction agents, suxamethonium, gentamicin and other antibiotics or low molecular weight heparins. It is, therefore, important to estimate weight when it cannot be measured directly.

In paediatric resuscitation, appropriate drug and fluid doses, equipment selection and ventilator settings are generally dependent on the weight of children. Because it is rarely possible to weigh a child in time-critical situations, it is customary to rely on a method of weight estimation and several tools have been developed for this purpose. Most of these are age-based or height-based and are less reliable with increasing age. ${ }^{12}$ Many drug and fluid

\section{Key messages}

What is already known on this subject? There are several methods to estimate paediatric weight in emergency or resource-poor situations when direct measurement is not possible, but there is no standard method of weight estimation in adults although many therapeutic interventions in adults are also based on weight. Mid-arm circumference has been used as a basis for paediatric weight estimation but it is not known whether this could be used in adolescents and adults.

\section{What this study adds?}

Using the US National Health and Nutrition Examination Survey, we have derived and validated a method of adolescent and adult weight estimation using mid-arm circumference. This study is based on pre-existing datasets and needs to be validated in a clinical setting.

regimens are weight dependent in all ages, but as yet there are no weight estimation tools for use in adults.

Age-based formulae (ABF) for paediatric weight estimation include those used in the APLS course. ${ }^{3}$ The APLS method uses two different formulae according to the age group of the child, as defined by the child's age last birthday: for children aged $1-5$ years, weight in $\mathrm{kg}=(2 \times$ age $)+8$. For children aged 6-12 years, weight in $\mathrm{kg}=(3 \times$ age $)+7$. Other formulae have also been used. ${ }^{4}$ However, none of these is reliable for children aged beyond 1012 years. Wide ranges of weights for adults of the same age preclude the use of $\mathrm{ABF}$ for adults.

The most commonly used height-based method of weight estimation is the Broselow tape (BT). The latest version of the tape extends to $150 \mathrm{~cm}$, as well as providing an estimate of weight according to the height of children, and classifies children into colour-coded groups corresponding to appropriate sets of equipment sizes and drug doses. ${ }^{5}$ However, the tape is unsuitable for use in most children aged over 10 years. These older children are either too tall to fit the dimensions of the tape, or if they do fit, their height does not correlate with weight as strongly as it does in younger children. ${ }^{6}$ As for $\mathrm{ABF}$, wide ranges of weight for height in adults will preclude the use of adult weight estimation methods based on height alone.

Mid-arm circumference (MAC) is widely used as an indicator of childhood nutrition status in 
resource-limited countries. WHO guidelines use a cut-off of $11.5 \mathrm{~cm}$ as one criterion in the diagnosis of severe acute malnutrition, and MAC is increasingly used in adolescents and adults too, especially in pregnant women and people living with $\mathrm{HIV}^{7}$ Although MAC has long been known to correlate with weight, no MAC-based weight estimation tool was published until 2010. This was a simple formula derived in children of Chinese school: weight in $\mathrm{kg}=(\mathrm{MAC}$ in $\mathrm{cm}-10) \times 3$. It performed as well as BT in older children, but poorly in preschool children. ${ }^{1}$ In 2012, the Mercy Tape was developed, which uses a combination of measurements of both MAC and humeral length to estimate weight, and was more precise than any other method in children aged $\leq 16$ years. $^{8} 9$

Our hypothesis was that MAC could provide the basis of an acceptable weight estimation tool for use in adults, adolescents and children. This study therefore aimed to derive and validate MAC-based formula for weight estimation for use in all age groups.

\section{METHODS \\ Data}

This study was an analysis of publicly available National Health and Nutrition Examination Survey (NHANES) datasets, downloaded from the CDC website. NHANES is a programme of studies designed to assess the health and nutritional status of adults and children in the USA. It combines interviews and physical examinations to collect health data for a nationally representative sample of the civilian population. Full details of the extremely in-depth sampling and examination methods are available on their website. ${ }^{10}$

We included all subjects aged over 12 months and extracted age and anthropometric data for each subject, including weight, height, MAC, upper arm length, upper leg length, waist circumference and sagittal abdominal diameter. Weight was measured digitally in kilograms, with subjects wearing a standard disposable gown and underwear. MAC was measured in the right arm at the midpoint between the tip of the olecranon and the acromion, with the arm hanging loosely.

We used the latest available NHANES dataset (2011-2012) for derivation.

\section{Statistical analysis}

LMS Chartmaker Pro V.2.3 software (Cole and Pan, Medical Research Council UK, 2006) was used to model the relationship of weight with MAC, according to the LMS method of Cole and Green. ${ }^{11}$ For each value (in $\mathrm{cm}$ ) of MAC, the model provides an estimate of the median weight in kilograms. Two models were constructed. One model used separate analyses for male and female to provide different median weights for each (gender specific). The other model used a gender-weighted analysis to provide single median weights for use in both male and female (gender neutral). MedCalc V. 14.12.0 (MedCalc Software bvba, Belgium) was used for all other analysis. Correlation of weight with each of the different body measurements was determined using Pearson's correlation coefficient, $r$, with 95\% CIs. Linear regression was used to define formulae relating weight with MAC.

\section{Model validation}

The NHANES 2009-2010 dataset was used as the validation set. Weight was estimated for all subjects according to the models developed in the derivation set. Bland-Altman analysis ${ }^{12}$ was used to determine the accuracy of these estimates. Accuracy can be described in terms of trueness and precision. Trueness is the measure of the average bias of the method in a given population. This is indicated by Bland-Altman bias, the mean percentage difference between estimated and actual weight. Precision is a measure of the spread of estimates around that mean. This is indicated by Bland-Altman limits of agreement (LOA), defined as $1.96 \times \mathrm{SD}$, the range within which $95 \%$ of the differences between estimated and actual weights will fall.

In addition, for each subject, the difference between the estimate of weight and the true weight was expressed as an absolute percentage error. The overall proportions of estimates with errors of $<10 \%, 20 \%$ and $30 \%$ were calculated for each method. The $\chi^{2}$ testing was used to compare these proportions between different methods.

Four age groups were defined as 1-5.9, 6-10.9, 11-15.9 and $\geq 16$ years. The previous MAC-weight formula was derived in children aged from 1 to 11 years, ${ }^{1}$ and the Mercy tape in children aged $\leq 16$ years. ${ }^{2}$ The age groups in this study were chosen to reflect those cut-offs, divided equally into 5 -year ranges.

\section{Ethics}

Ethical approval was not sought as the data were already publicly available online. No subjects were further involved, and no patient identifiable data were obtained. There was also no funding source for this project.

\section{RESULTS}

Data

A total of 8498 subjects (50.1\% male) were included from the NHANES 2011-2012 dataset; 5595 adults (aged 16-80 years) and 2903 children (aged 1-15.9 years). The ethnicity of the dataset reflected that of the US population.

The population distributions of the NHANES datasets were assessed in terms of gender, age, MAC and weight. Derivation (2011-2012) and validation (2009-2010) datasets are presented in table 1 . There was no significant difference in gender proportion $\left(p=0.69, \chi^{2}\right.$ test $)$ as well as in mean age $(p=0.40$, t-test). There were small differences in mean $\operatorname{MAC}(0.3 \mathrm{~cm}, \mathrm{p}=0.01$, t-test) and weight $(1.3 \mathrm{~kg}, \mathrm{p}=0.005$, t-test).

\section{Statistical analysis}

Correlation of each body measurement with weight is shown in table 2. In both adults and children, the correlation of weight was significantly stronger with MAC (overall; $r=0.96,95 \% \mathrm{CI}$ 0.96 to 0.96 ) than with the next best, waist circumference $(\mathrm{r}=0.95,95 \%$ CI 0.95 to 0.95$)$.

The relationship of MAC with weight was nearly linear (figure 1), with non-linearity at lower values of MAC (figure 2). Using the LMS method to model the data for best fit, median

Table 1 Descriptive statistics of key variables

\begin{tabular}{|c|c|c|c|c|}
\hline \multirow{4}{*}{$\begin{array}{l}\text { n } \\
\text { Male (\%) }\end{array}$} & \multirow{2}{*}{\multicolumn{2}{|c|}{$\begin{array}{l}\text { Derivation dataset (NHANES } \\
\text { 2011-2012) } \\
8498\end{array}$}} & \multicolumn{2}{|c|}{$\begin{array}{l}\text { Validation dataset (NHANES } \\
\text { 2009-2010) }\end{array}$} \\
\hline & & & 9022 & \\
\hline & \multicolumn{2}{|c|}{$4240(50.1 \%)$} & \multicolumn{2}{|c|}{$4484(49.7 \%)$} \\
\hline & Mean (SD) & Median (IQR) & Mean \pm SD & Median (IQR) \\
\hline Age (years) & $32.3 \pm 23.8$ & $29(11-52)$ & $32.0 \pm 22.9$ & $29(11-51)$ \\
\hline Weight (kg) & $64.0 \pm 30.3$ & $66.1(43.4-83.7)$ & $65.3 \pm 31.0$ & $68(44.7-85.4$ \\
\hline MAC (cm) & $28.8 \pm 7.6$ & $39.6(23.4-34.2)$ & $29.1 \pm 7.7$ & $30.1(23.8-34.5$ \\
\hline
\end{tabular}

All results are rounded to one decimal place.

MAC, mid-arm circumference; NHANES, National Health and Nutrition Examination Survey. 
Table 2 Correlation of weight with other body measurements

\begin{tabular}{|c|c|c|c|}
\hline & Overall & $\begin{array}{l}\text { Adults } \\
\text { ( } \geq 16 \text { years) }\end{array}$ & $\begin{array}{l}\text { Children } \\
\text { (1-15.9 years) }\end{array}$ \\
\hline MAC & $0.96(0.96$ to 0.96$)$ & $0.90(0.90$ to 0.91$)$ & $0.96(0.96$ to 0.97$)$ \\
\hline Height & $0.80(0.79$ to 0.81$)$ & $0.42(0.40$ to 0.44$)$ & 0.87 (0.86 to 0.88$)$ \\
\hline Upper arm length & $0.86(0.85$ to 0.86$)$ & $0.59(0.57$ to 0.60$)$ & $0.90(0.89$ to 0.91$)$ \\
\hline Upper leg length & $0.46(0.44$ to 0.48$)$ & $0.29(0.27$ to 0.32$)$ & $0.75(0.72$ to 0.77$)$ \\
\hline Waist circumference & $0.95(0.95$ to 0.95$)$ & $0.89(0.89$ to 0.90$)$ & $0.94(0.94$ to 0.95$)$ \\
\hline Abdominal diameter & $0.89(0.89$ to 0.90$)$ & $0.86(0.85$ to 0.86$)$ & 0.87 (0.86 to 0.89$)$ \\
\hline
\end{tabular}

values of weight were derived for each value of MAC. These are presented in online supplementary table S1, with median weights overall, and for each gender. From linear regression of the whole sample, weight in $\mathrm{kg}=(3.8484 \times \mathrm{MAC}$ in $\mathrm{cm})$ -46.8585 . The coefficients of this equation were rounded to one significant figure to provide a simplified version of this equation: weight in $\mathrm{kg}=(4 \times \mathrm{MAC})-50$.

We therefore tested four alternative MAC-based weight estimation methods in the validation set:

- Method A: the gender neutral LMS model

- Method B: the gender specific LMS model

- Method C: the exact linear regression equation

- Method D: the simplified equation

\section{Model validation}

A total of 9022 subjects $(49.8 \%$ male) were included from the NHANES 2009-2010 dataset for validation; 6049 adults (aged 16-79 years) and 2973 children (aged 1-15.9 years). Three subjects had MAC measurements that were outside the limit of the derived model $(13-58 \mathrm{~cm})$ and were not included in the analysis of the LMS method.

Bland-Altman results for percentage bias and LOA are given in table 3, together with the percentage of estimates falling within $10 \%, 20 \%$ and $30 \%$ of actual weight. Examples of Bland-Altman plots are given for all methods in adults in figure 3 .

In the set of all subjects, Bland-Altman analysis demonstrated biases of approximately $1 \%$ overestimation. LOA ranged from approximately $\pm 26 \%$ (method B) to $\pm 31 \%(\operatorname{method} \mathrm{D})$ around the bias.

Overall, in terms of the proportions of estimates within $\mathrm{x} \%$ of actual weight, method $\mathrm{B}$ performed best and method D worst. However, the differences were small and most did not reach significance. Differences between methods D and C only reached significance for estimates within $20 \%\left(p=0.03, \chi^{2}\right.$ test), between methods $\mathrm{C}$ and A only for estimates within $30 \%$ $\left(p=0.04, \chi^{2}\right.$ test) and between methods A and B only for estimates within $10 \%$ ( $p=0.02, \chi^{2}$ test).

Table 3 presents results according to age groups. In adults and children aged 11-15.9 years, biases were generally small (all within $\pm 2.5 \%$ ) and LOA narrow (all lying between $\pm 20 \%$ and $\pm 25 \%$ around the bias). In all models, at least $60 \%, 90 \%$ and $98 \%$ of estimates fell, respectively, within $10 \%, 20 \%$ and $30 \%$ of actual weights for adults and adolescents. In comparing proportions of estimates within $20 \%$ of actual weight, results for all models were slightly better in adults compared with children aged 11-15.9 years, but this was significant only for methods $\mathrm{A}$ and $\mathrm{B}(\operatorname{method} \mathrm{A}, \mathrm{p}=0.007$; method $\mathrm{B}, \mathrm{p}<0.0001$; method $\mathrm{C}$, $\mathrm{p}=0.12$; method $\mathrm{D}, \mathrm{p}=0.46$; all with $\chi^{2}$ test).
However, results were much worse in children aged 610.9 years and still worse in those aged 1-5.9 years. Similar patterns were found on subgroup analysis according to MAC or weight: all methods performed poorly in subjects with MAC $<20 \mathrm{~cm}$ or weight $<30 \mathrm{~kg}$.

\section{DISCUSSION}

In the prehospital setting and in resuscitation in-hospital, weighing patients directly is not feasible, unless trolleys with sophisticated built-in scales are available. Until these are standards, there will be a need for weight estimation in emergency situations. The need to estimate weight of patients is more important in low/ middle-income countries, where there might be less access to calibrated scales in less emergent environments as well. ${ }^{2}$

This study has demonstrated that in the NHANES datasets, weight is strongly correlated with MAC in adults as well as in children and more so than with other body measurements. The next best parameter is waist circumference, but it would be impractical to use this as a method to estimate weight in emergency situations, where the patient is usually in the supine position. The relationship of MAC with weight is very nearly linear and more so with increasing MAC. This suggests that a linear weight estimation method could be especially appropriate in adults.

We have demonstrated the trueness and precision of linear regression equations relating MAC with weight, in comparison with more complex modelling methods relying on the LMS method of best fit. The simplified equation, weight in $\mathrm{kg}=(4 \times$ $\mathrm{MAC})-50$, could be worked out without recourse to calculators. The LMS gender-specific rule would require a smartphone application or a specially designed tape measure. Both tools are less precise in children than in adults and adolescents, and we do not recommend their use for patients aged below 11 years. The simplified equation is significantly less precise than the LMS gender-specific model, but we consider that when used in adolescents or adults, both are well within the level of what is considered adequate for use in clinical practice.

Unfortunately, there is neither any agreed standard to determine the clinical acceptability of the accuracy of a weight estimation method nor are there any other weight estimation tools for use in adults to make a direct comparison with. Instead, we need to consider the accuracy of methods currently accepted for use in children. Unfortunately, there is little consistency in the way accuracy is described in different studies, and it is difficult to make direct comparisons. Bland-Altman analysis (or similarly, mean percentage error $\pm S D$ ) has been used more recently, but not in early studies of BT or ABF. Proportions of estimates within $10 \%$ of actual weights were described in the original BT 
Figure 1 Distribution of mid-arm circumference (MAC) with weight.

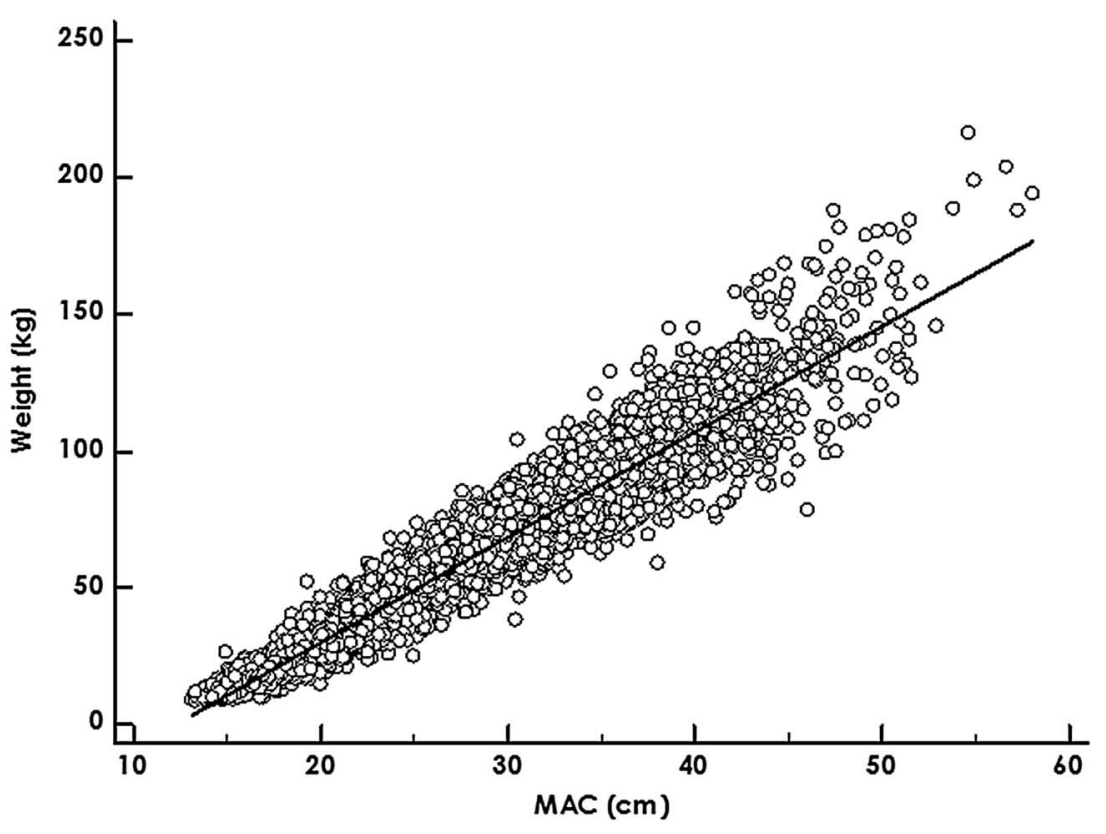

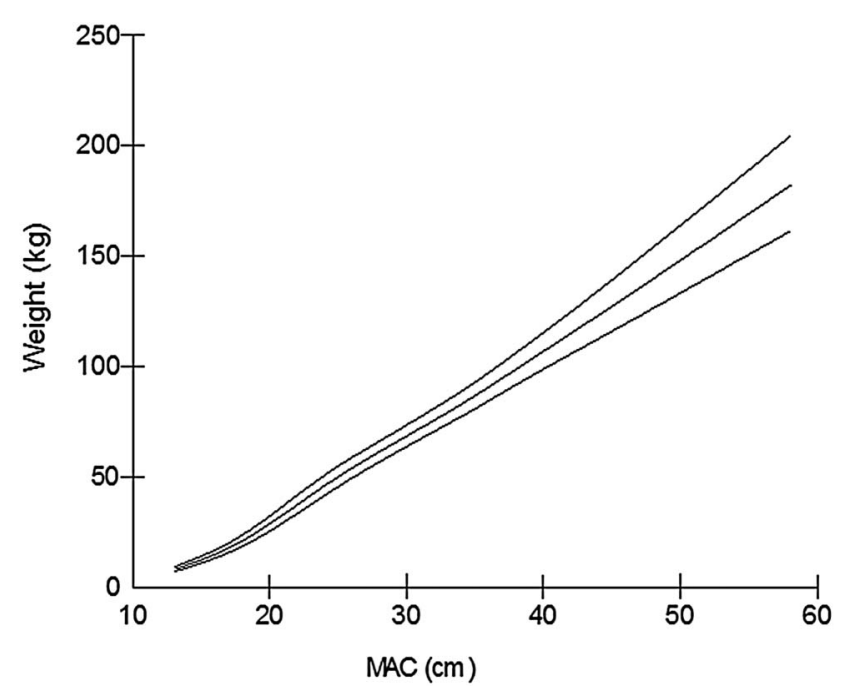

Figure 2 LMS model of MAC with weight: median values and interquartile ranges.

paper, ${ }^{5}$ but those authors have subsequently doubted whether we really need to be that precise, ${ }^{13}$ and other studies of weight estimation have used wider ranges. ${ }^{1}{ }^{2}$ Different drugs have different therapeutic ranges, and the degree of precision necessary will vary. Drugs with very narrow therapeutic ranges are unlikely to be required in the sort of time-critical or resourcepoor contexts that necessitate weight estimations. In adult medicine, most drugs are given as a single adult dose, and even a very conservative range of adult weight from 50 to $100 \mathrm{~kg}$ implies an equivalent precision of $\pm 33 \%$ around an average $75 \mathrm{~kg}$.

There appears to be little evidence that patients are adversely affected by $10 \%-30 \%$ errors of actual weight. However, the absence of such an effect may be because it has not been investigated rather than that there is no effect. Second, we believe that most physicians in emergency settings would aim to estimate weights within $10 \%$ of actual weight and would be uncomfortable if their estimates were more than $30 \%$ out. It is beyond the scope of this study to determine the precision actually needed for different drugs. We therefore chose to present proportions of estimates within $10 \%, 20 \%$ and $30 \%$ of actual weight to demonstrate how precisely one can estimate weight in adults.

The original BT paper studied 937 children, with a skew towards younger age groups. ${ }^{5}$ Note that, $59.7 \%$ of estimates were within $10 \%$ of actual weight. Since then, several studies in different populations have found similar results, ranging from $53 \%$ to $65 \%$, with the best results in preschool children. ${ }^{1}{ }^{14-17}$ Trueness is often dependent on the population studied, ${ }^{18}$ with bias (mean error) ranging from $11.3 \%$ overestimation in India ${ }^{19}$ to $11.9 \%$ overestimation in Canada. ${ }^{20}$ The increasing weight of children in America has necessitated several modifications of the tape to avoid underestimation in that setting. In the original paper, BT underestimated by nearly $0.4 \mathrm{~kg}$ in children weighing $10-25 \mathrm{~kg}$ (approximately 2\%-4\%), but by over $3 \mathrm{~kg}$ (approximately $10 \%$ ) in those heavier. ${ }^{5}$ Levels of agreement can range from approximately $\pm 25 \%$ in younger children to as much as $\pm 50 \%$ in older children. ${ }^{1} 1421$

Age-based methods are imprecise, although some may have minimal bias. The recent change in the APLS formula was an attempt to correct underestimation with the previous formula. It is mathematically straightforward to create an $\mathrm{ABF}$ with nearly zero bias, simply by using the median weight for each year of age. However, the LOA are always larger than with BT, and fewer estimates lie within $10 \%$ of true. In other words, trueness can be fine-tuned, but the inherent imprecision remains. For example, in a recent study comparing 12 different ABF, biases ranged from $-10.1 \%$ to $+19.7 \%$, but in all cases LOA were approximately $\pm 40 \%$ around the bias. The best rules estimated fewer than $90 \%$ of children within $30 \%$ of actual weight. Precision decreased with increasing age. ${ }^{4}$ Other studies have found similar results, with only about $40 \%$ of estimates within $10 \%$ of actual weight. ${ }^{15} 22$

Two newer methods, the PAWPER tape ${ }^{14}$ and the Mercy tape, ${ }^{8}$ have been shown to estimate, respectively, nearly $90 \%$ and $80 \%$ of children to within $10 \%$ of actual weight. The PAWPER tape is a height-based method modified for body habitus; the Mercy tape uses a combination of MAC and humeral length. However, none of them is widely used yet and 
Table 3 Accuracy of weight estimation methods

\begin{tabular}{|c|c|c|c|c|c|c|}
\hline \multirow[b]{2}{*}{ Age (years) } & \multirow[b]{2}{*}{$\mathbf{n}$} & \multicolumn{2}{|c|}{ Bland-Altman analysis } & \multicolumn{3}{|c|}{ Percentage of estimates lying within $\mathrm{x} \%$ of actual weight } \\
\hline & & Bias (\%) & LOA (\%) & Within $10 \%$ & Within $20 \%$ & Within $30 \%$ \\
\hline \multicolumn{7}{|l|}{ Method A } \\
\hline All ages & 9019 & 0.9 & -25.9 to 27.6 & 58.6 & 86.8 & 95.4 \\
\hline $1-5.9$ & 1169 & 11.2 & -23.1 to 45.4 & 37.2 & 63.7 & 78.8 \\
\hline $6-10.9$ & 961 & 2.5 & -32.5 to 37.4 & 38.6 & 70.3 & 90.2 \\
\hline $11-15.9$ & 843 & -2.3 & -27 to 22.4 & 63.0 & 90.7 & 98.0 \\
\hline $16+$ & 6046 & -1 & -22.5 to 20.6 & 65.2 & 93.3 & 99.0 \\
\hline \multicolumn{7}{|l|}{ Method B } \\
\hline All ages & 9019 & 0.8 & -25.4 to 27.1 & 60.3 & 87.7 & 95.4 \\
\hline $1-5.9$ & 1169 & 11.3 & -22.8 to 45.5 & 36.9 & 62.4 & 78.0 \\
\hline $6-10.9$ & 961 & 2.6 & -32.1 to 37.4 & 38.9 & 71.1 & 89.4 \\
\hline $11-15.9$ & 843 & -2.3 & -27.1 to 22.5 & 65.0 & 90.5 & 98.0 \\
\hline $16+$ & 6046 & -1 & -21.6 to 19.5 & 67.5 & 94.9 & 99.3 \\
\hline \multicolumn{7}{|l|}{ Method C } \\
\hline All ages & 9022 & 0.4 & -28.4 to 29.3 & 57.7 & 85.8 & 94.7 \\
\hline $1-5.9$ & 1169 & 6 & -44.5 to 56.5 & 29.7 & 53.9 & 72.8 \\
\hline 6-10.9 & 961 & 4.4 & -28.3 to 37.1 & 42.4 & 71.9 & 90.5 \\
\hline $11-15.9$ & 843 & -2.5 & -25.7 to 20.7 & 63.1 & 91.9 & 98.6 \\
\hline $16+$ & 6049 & -0.8 & -22.4 to 20.7 & 64.8 & 93.4 & 99.1 \\
\hline \multicolumn{7}{|l|}{ Method D } \\
\hline All ages & 9022 & 1.3 & -29.5 to 32.1 & 56.6 & 84.7 & 94.1 \\
\hline $1-5.9$ & 1169 & 0.8 & -57.4 to 59 & 28.5 & 54.1 & 71.7 \\
\hline $6-10.9$ & 961 & 4 & -31.5 to 39.5 & 41.2 & 69.5 & 88.3 \\
\hline $11-15.9$ & 843 & -1.2 & -25.3 to 23 & 63.5 & 91.3 & 98.5 \\
\hline $16+$ & 6049 & 1.4 & -20.4 to 23.1 & 63.5 & 92.1 & 98.7 \\
\hline
\end{tabular}
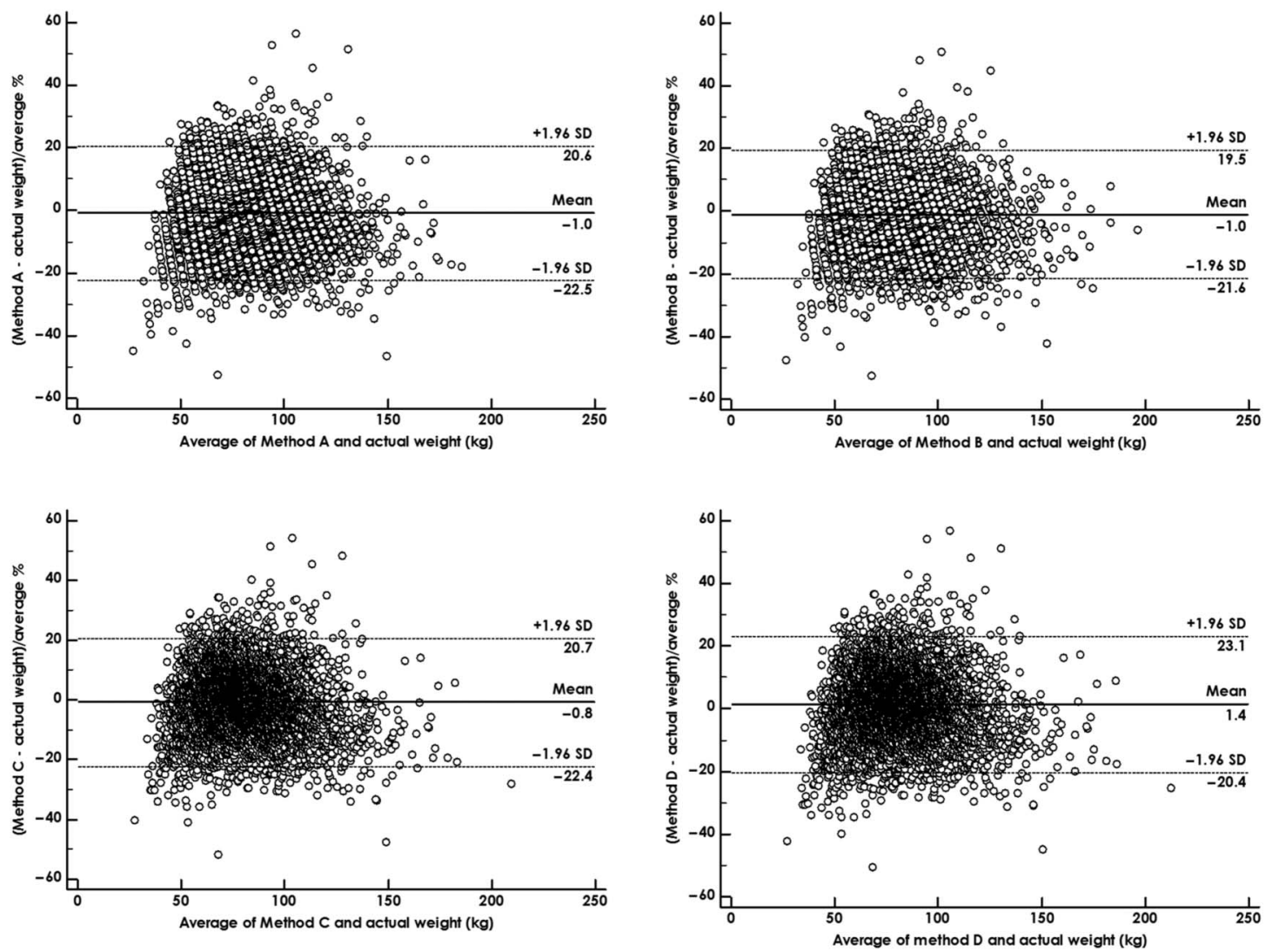

Figure 3 Bland-Altman plot for each method (A-D) in adults. 
both are designed only for children or adolescents. It is still considered acceptable practice to make treatment decisions based on weight estimations in children using methods with the accuracy outlined above.

We have demonstrated Bland-Altman biases and LOA in adults and adolescents that are smaller and narrower than accepted for both BT and ABF in children. Even with our least precise method, the simplified formula, the proportions of estimates in adults within $10 \%, 20 \%$ and $30 \%$ of actual weight $(63.5 \%, 92.1 \%$ and $98.3 \%)$ are at least as good as published results for BT and far better than for any ABF in children. Our gender-specific tool is significantly better still.

\section{Limitations}

We did not include infants, as we have previously shown that MAC-based formulae are unreliable in smaller children. ${ }^{1}$ This study confirmed that the MAC formula was not suitable in young children, and we do not recommend its use in children aged below 11 years.

The study was derived and validated in existing datasets, relying on measurements made by the NHANES investigators. It is possible that the measurement of MAC in real patients, particularly in emergency situations, would not be performed as effectively as in the NHANES study. This would be likely to make the method less precise. The use of this MAC formula to estimate weight needs to be validated in real patients, in different clinical settings including the ED.

The formula is based on US data (including representative proportions of people of different ethnicity) and should be validated in other countries. However, it should be noted that the BT is also based on the NHANES database and is used internationally nonetheless.

This paper does not address the question of whether actual weight is necessarily the most appropriate measure for drug and fluid dosing. In obesity or oedema, lean body weight might be more suitable (eg, in chemotherapy). However, in time-critical contexts, there is no doubt that if the actual weight were known, that is what would be used to determine doses. It is, therefore, entirely appropriate that estimates of weight should be compared with actual weight rather than the more complex concept of lean body weight.

\section{CONCLUSION}

Our study aimed to derive and validate a MAC-based formula for use in all age groups. We do not think the models we have derived are precise enough for use in children aged below 11 years. However, this method of weight estimation in adults is at least as precise as commonly used paediatric weight estimation tools. The simplified formula is appropriate for weight estimation in both adults and adolescents. Improved precision would be obtained with the gender-specific model, and this would require a specialised tape or smartphone application. These MAC-based methods could provide an accurate, cheap and simple method of weight estimation in many settings for most patients.

Contributors All authors contributed to the original idea for the paper, and the writing and revision of it. GNC performed the data analysis and wrote the original draft.

Competing interests None declared.
Provenance and peer review Not commissioned; externally peer reviewed.

Data sharing statement All data used for this study are open access, from the NHANES website.

Open Access This is an Open Access article distributed in accordance with the Creative Commons Attribution Non Commercial (CC BY-NC 4.0) license, which permits others to distribute, remix, adapt, build upon this work non-commercially, and license their derivative works on different terms, provided the original work is properly cited and the use is non-commercial. See: http://creativecommons.org/ licenses/by-nc/4.0/

\section{REFERENCES}

1 Cattermole GN, Leung PYM, Mak PSK, et al. Mid-arm circumference can be used to estimate children's weights. Resuscitation 2010;81:1105-10.

2 Abdel-Rahman SM, Ridge A, Kearns GL. Estimation of body weight in children in the absence of scales: a necessary measurement to insure accurate drug dosing. Arch Dis Child 2014;99:570-4.

3 Advanced Life Support Group. Advanced paediatric life support-the practical approach. 5th edn. Oxford: Wiley-Blackwell, 2011.

4 Cattermole GN, Leung MPY, So HK, et al. Age-based formulae to estimate children's weight in the emergency department. Emerg Med J 2011;28:390-6.

5 Lubitz DS, Seidel JS, Chameides L, et al. A rapid method for estimating weight and resuscitation drug dosages from length in the pediatric age group. Ann Emerg Med 1988; 17:576-81.

6 Cattermole GN, Leung PYM, Graham CA, et al. Too tall for the tape: the weight of schoolchildren who do not fit the Broselow tape. Emerg Med J 2014;31:541-4.

7 Tang AM, Dong K, Deitchler M, et al. Use of cutoffs for mid-upper arm circumference (MUAC) as an indicator or predictor of nutritional and health-related outcomes in adolescents and adults: a systematic review. Washington: FANTA, 2013.

8 Abdel-Rahman SM, Ridge AL. An improved pediatric weight estimation strategy. Open Med Devices J 2012:4:87-97.

9 Abdel-Rahman SM, Paul IM, James LP, et al. Evaluation of the Mercy TAPE: performance against the standard for pediatric weight estimation. Ann Emerg Med 2013:62:332-9.

10 Centers for Disease Control and Prevention (CDC). National Center for Health Statistics (NCHS). National Health and Nutrition Examination Survey Data. Hyattsville, MD: U.S. Department of Health and Human Services, Centers for Disease Control and Prevention, 1999-2008. http://www.cdc.gov/nchs/nhanes/ nhanes_questionnaires.htm

11 Cole TJ, Green PJ. Smoothing reference centile curves: the LMS method and penalized likelihood. Stat Med 1992;11:1305-19.

12 Altman DG, Bland JM. Measurement in medicine: the analysis of method comparison studies. Statistician 1983;32:307-17.

13 Luten RC, Zaritsky A, Wears R, et al. The use of the Broselow tape in pediatric resuscitation. Acad Emerg Med 2007;14:500-1.

14 Wells M, Coovadia A, Kramer E, et al. The PAWPER tape: a new concept tape-based device that increases the accuracy of weight estimation in children through the inclusion of a modifier based on body habitus. Resuscitation 2013; 84 227-32.

15 Krieser D, Nguyen K, Kerr D, et al. Parental weight estimation of their child's weight is more accurate than other weight estimation methods for determining children's weight in an emergency department? Emerg Med J 2007;24:756-9.

16 Nieman CT, Manacci CF, Super DM, et al. Use of the Broselow tape may result in the underresuscitation of children. Acad Emerg Med 2006;13:1011-19.

17 Hofer CK, Ganter M, Tucci M, et al. How reliable is length-based determination of body weight and tracheal tube size in the paediatric age group? The Broselow tape reconsidered. Br J Anaesth 2002:88:283-5.

18 House DR, Ngetich E, Vreeman RC, et al. Estimating the weight of children in Kenya: do the Broselow Tape and age-based formulas measure up? Ann Emerg Med 2013:61:1-8.

19 Ramarajan N, Krishnamoorthi R, Strehlow M, et al. Internationalizing the Broselow tape: how reliable is weight estimation in Indian children. Acad Emerg Med 2008; 15:431-6

20 Bourdeau S, Copeland J, Milne WK. Accuracy of the Broselow tape in estimating the weight of First Nations children. Can J Rural Med 2011;16:121-5.

21 DuBois D, Baldwin S, King WD. Accuracy of weight estimation methods for children. Pediatr Emerg Care 2007;23:227-30.

22 Nguyen K, Krieser D, Kerr D, et al. Failed validation of the Argall weight formula for estimating children's weight in an Australian emergency department. Acad Emerg Med 2007;14:486-8. 